\title{
Femtosecond laser functionalized surfaces inspired by nature (Conference Presentation) (Withdrawal Notice)
}

Camilo Florian Baron, Javier Solis, Jan Siegel, Jörg Krüger, Jörn Bonse

Camilo Florian Baron, Javier Solis, Jan Siegel, Jörg Krüger, Jörn Bonse, "Femtosecond laser functionalized surfaces inspired by nature (Conference Presentation) (Withdrawal Notice)," Proc. SPIE 11268, Laser-based Microand Nanoprocessing XIV, 112680J (27 March 2020); doi: 10.1117/12.2542308

SPIE. Event: SPIE LASE, 2020, San Francisco, California, United States 


\section{Femtosec ond laser func tionalized surfaces inspired by nature (Conference Presentation) (Withdrawal Notice)}

Camilo Forian Baron, J örg Krüger, Jöm Bonse, Bundesansta It für Materialforschung und -prüfung (Germany) Javier Solis, J an Siegel, Instituto de Óptic a "Daza de Valdés" (Spain)

Proceedings Volume 11268, Laser-based Micro- and Nanoprocessing XIV; 112680J

(2020) https://doi.org/10.1117/12.2542308

Event Event: SPIE LASE, 2020, San Francisc 0, Califomia, United Sta tes

Online Publication Date: 9 March 2020

Withdrawn from Publication: 25 March 2020

Publisher's Note: This Conference Presentation, origina lly published on 9 March 2020, was withd rawn per author request. 